\title{
Expression of estrogen and progesterone receptors and Ki-67 antigen in Graves' disease and nodular goiter
}

\author{
Pawel Domoslawski' ${ }^{1}$, Bartosz Pula², Tadeusz Lukienczuk ${ }^{1}$, \\ Marzenna Podhorska-Okolow ${ }^{2}$, Piotr Dziegiel ${ }^{2,3}$
}

${ }^{1}$ Department of General, Gastroenterological and Endocrinological Surgery, Medical University of Wroclaw

${ }^{2}$ Department of Histology and Embryology, Medical University of Wroclaw

${ }^{3}$ Department of Physiotherapy, Wroclaw University School of Physical Education, Wroclaw, Poland

\begin{abstract}
Graves' Disease (GD) is an autoimmune disease with higher prevalence in women than in men. The aim of the study was to correlate the expression of estrogen (ER) and progesterone receptors (PR) as well as Ki67 cell proliferation index in thyroid sections of GD and nodular goiter (ND) patients. The study was performed on archival paraffin blocks of 77 GD and 25 ND patients using immunohistochemistry. Higher expression of progesterone receptors $(\mathrm{p}=0.0276)$ and Ki-67 index $(\mathrm{p}<0.0001)$ was observed in thyroids of GD as compared to NG patients. No correlations were found between the particular markers and patients' clinicopathological parameters, sex or age. A higher incidence of GD in women was not associated with different thyroid expression of ER and PR in comparison to men. No correlation was found between the expression of ER and PR and proliferation marker of thyrocytes of GD and ND patients. Significantly higher expression of the Ki-67 antigen in GD lesions was observed as compared to the NG. (Folia Histochemica et Cytobiologica 2013, Vol. 51, No. 2, 135-140)
\end{abstract}

Key words: thyrocytes, Graves' disease, nodular goiter, estrogen receptors, progesterone receptors, Ki-67

\section{Introduction}

Graves' Disease (GD) is a common organ-specific autoimmune disorder of recurrent nature and complex pathogenesis involving genetic and environmental factors resulting in majority of the cases in hyperthyroidism [1]. Autoimmune diseases (AID) appear when the host immune system turns against its own antigens leading to dysfunction or destruction of tissues and organs. AID may develop in mechanisms involving immune deregulation, genetic predisposition and due to influence of environmental factors [2]. Thyroid autoimmune diseases like GD and Hashimoto thy-

\footnotetext{
Correspondence address: P. Domoslawski,

Department of General, Gastroenterological and Endocrinological Surgery,

Medical University of Wroclaw;

Sklodowskiej-Curie St. 66, 50-369 Wroclaw Poland;

tel.: +48 71784 2162; fax: +48 717959619 ;

e-mail: pawel.domoslawski@umed.wroc.pl
}

roiditis (HT) affect the thyroid gland and are called autoimmune thyroid diseases (AITD). They are complex diseases caused by an interaction of susceptibility genes and other, non-genetic factors like infection [1, 3]. The background of GD appears to have a strong genetic nature associated with the polymorphism in the HLA, CTLA-4 (cytotoxic T-lymphocyte associated antigen-4) and protein tyrosine phosphatase type 22 (PTPN22) genes but the pathogenesis is still not clear [1]. In GD thyroid-reactive T cells infiltrate the thyroid gland and activate B cells to produce thyrotropin receptor (TSHR) stimulating autoantibodies, leading to stimulation of thyroid follicular cells [3]. As a result, the negative control feedback loop of the pituitary system is superseded by the stimulating antibodies leading to constant thyrocyte activation via the TSHR and the production of thyroid hormones, thyroxine $\left(\mathrm{T}_{4}\right)$ and triiodothyronine $\left(\mathrm{T}_{3}\right)$ leading to hyperthyroidism [3].

Like other autoimmune diseases, GD appears more frequently in women than in men at a ratio of 
$7: 1$, which might suggest the role of hormonal factors in its pathogenesis [4]. Recent data showed that women are up to 7-10 times more likely susceptible to have GD, systemic lupus erythematous or Sjogren's disease [5]. On the other hand, some other autoimmune diseases, e.g. rheumatoid arthritis or multiple sclerosis develop only 2-3 times more often in women than in men [5]. When unanticipated dimorphic findings appear between sexes, the first consideration is given to sex steroid hormones, which are known as potent mediators for sex differences. Sex steroid hormones include estrogens, progesterone and androgens and their action is dependent upon their circulating blood levels and expression of congruent hormone receptors [5].

The protein, Ki-67 antigen is widely used as a marker of cell proliferation. Its precise function in cell proliferation is still unknown [6-10]. It was found that Ki-67 may be involved in perinuclear chromatin organization and its highest expression is noted in late G1, S and $G 2$ phase of the cell cycle $[6,11]$. In addition to its expression in proliferating cells, $\mathrm{Ki}-67$ expression was also noted in processes associated with inhibited DNA synthesis or in the course of apoptosis $[6,11]$. Despite some incoherent information concerning the biological significance of Ki-67 in cell physiology, this antigen is being currently routinely used as marker of cell proliferation in clinicopathological diagnostic methods, therefore it may also serve as a potent proliferation marker of thyroid follicular cells [11-13].

The purpose of this retrospective study was to identify the association between the expression of estrogen receptors (ER), progesterone receptors (PR) in patients with GD to determine if these factors have influence on GD and its higher incidence in women. Moreover, the study aimed at examining the effects of ER and PR on proliferation rate of thyrocytes of GD patients, by assessing the expression of the Ki-67 antigen.

\section{Material and methods}

Patients and tissue specimens. The study was performed on archival paraffin blocks of 77 patients with diagnosed GD and 25 patients with nodular goiter (ND), who were operated on at the Department of General, Gastroenterological and Endocrinological Surgery of Wroclaw Medical University in the years 2003-2007. The mean age of patients with Graves' disease was $38.89 \pm 12.68$ (range 16-68) years, whereas nodular goiter patients were aged $40.28 \pm 12.1$ (range 21-61) years. In case of GD, 15 (19.5\%) patients were male, whereas $62(80.5 \%)$ were of female sex. In the NG group $6(24.0 \%)$ patients were male and 19 (76.0\%) were female. The study was approved by the Bioethical Commission of the Wroclaw Medical University.
Histopathological examination and immunohistochemical reactions (IHC). Tissue samples were fixed in 10\% buffered formalin, dehydrated and embedded in paraffin blocks. For histopathological examination, the 6- $\mu \mathrm{m}$ sections were stained with hematoxylin and eosin (H\&E) and reviewed by a pathologist to verify the diagnosis. Immunohistochemical reactions were performed on 4- $\mu \mathrm{m}$-thick sections mounted on Superfrost Plus slides (Menzel Gläser, Braunschweig, Germany), deparaffinized in xylene and gradually rehydrated. Then, the sections were boiled in Target Retrieval Solution buffer (pH 6, 20 min; DakoCytomation, Glostrup, Denmark), subsequently cooled in distillate water and $1 \times$ PBS. Sections were then incubated with $3 \%$ hydrogen peroxide for $5 \mathrm{~min}$ to block the endogenous peroxidase. Next, the sections were incubated with primary monoclonal antibodies directed against ER (clone 1D5, ready-to-use, specific for detecting only the type), PR (clone PgR 636, ready-to-use) and Ki-67 (clone MIB1, ready-to-use) for $45 \mathrm{~min}$ in room temperature (DakoCytomation). The antigens were visualized using biotinylated antibodies and streptavidin conjugated with horseradish peroxidase (EnVision ${ }^{\mathrm{TM}}$ Mouse HRP, DakoCytomation). Diaminobenzidine (DAB, DakoCytomation) was used as the substrate. All the reactions were conducted using negative controls and all the slides were counterstained with hematoxylin (DakoCytomation).

Evaluation of IHC reactions. The IHC sections were evaluated using BX-41 light microscope (Olympus, Tokyo, Japan) by two independent pathologists who were blinded to patients' clinical data. In doubtful cases a re-evaluation was performed using a double-headed microscope and the staining was discussed until a consensus was achieved. ER, PR and Ki-67 antigen expression in follicular cells was assessed in whole tissue sections using a semiquantitative five-point scoring system-based cell positivity. It was encoded as follows: 0 ( $0 \%$ cells stained), 1 (1-5\% cells stained), 2 (6-10\% cells stained), 3 (11-20\% cells stained), 4 (21-100\% cells stained).

Statistical analysis. The obtained results were subjected to statistical analysis using Prism 5.0 (GraphPad, La Jolla, US). The Shaphiro-Wilk normality test was used to assess the normality of the data distribution. Correlation between expression intensities of selected markers was examined using Spearman correlation test. The Mann-Whitney U test was applied to compare the differences in expression of the studied markers between the groups. The results were considered significant at $\mathrm{p}<0.05$.

\section{Results}

Nuclear expression of ER, PR and Ki-67 was observed in thyrocytes of patients with GD and compared to their expression in patients with NG (Figure 1). The 


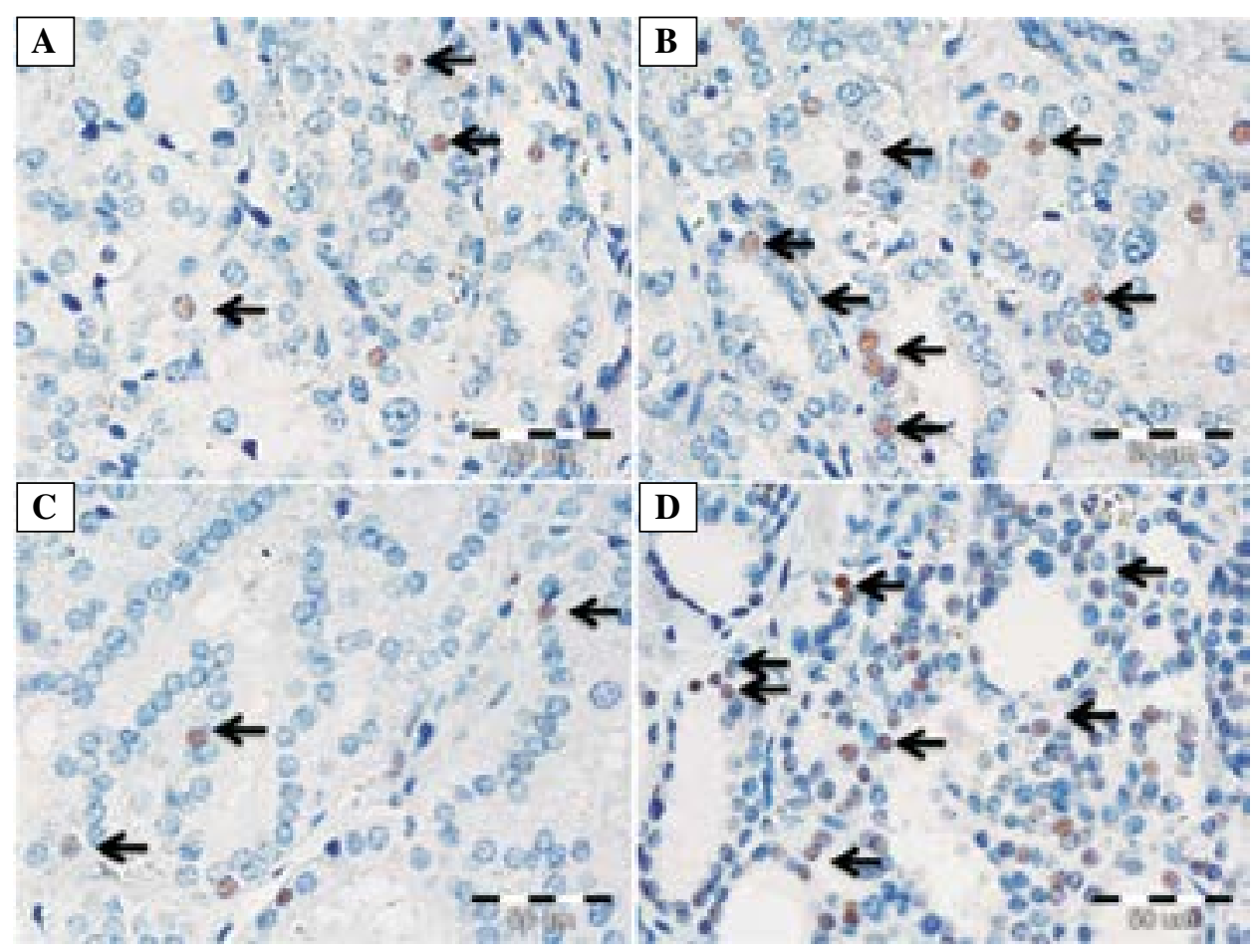

Figure 1. Low (A, C) and high (B, D) nuclear expression of PR $(\mathbf{A}, \mathbf{B})$ and ER $(\mathbf{C}, \mathbf{D})$ in thyroids of Graves' disease patients. Arrowheads indicate positive nuclear reaction. Magnification $\times 400$

Table 1. Distribution of ER, PR and Ki-67 in thyrocytes of Graves' disease and nodular goiter patients

\begin{tabular}{|l|l|l|l|}
\hline IHC marker score & Graves' disease No. cases (\%) & Nodular goiter No. cases (\%) & $\begin{array}{l}\text { All patients } \\
\text { No. cases (\%) }\end{array}$ \\
\hline ER & \multicolumn{2}{l|}{} \\
\hline $0(0 \%)$ & $36(46.7)$ & $8(32.0)$ & $44(43.1)$ \\
\hline $1(1-5 \%)$ & $19(24.7)$ & $12(48.0)$ & $31(30.4)$ \\
\hline $2(6-10 \%)$ & $12(15.6)$ & $4(16.0)$ & $16(15.7)$ \\
\hline $3(11-20 \%)$ & $6(7.8)$ & $1(4.0)$ & $7(6.9)$ \\
\hline $4(21-100 \%)$ & $4(5.2)$ & $0(0.0)$ & $4(3.9)$ \\
\hline PR & \multicolumn{3}{l|}{} \\
\hline $0(0 \%)$ & $32(41.6)$ & $15(60.0)$ & $47(46.1)$ \\
\hline $1(1-5 \%)$ & $16(20.8)$ & $7(28.0)$ & $23(22.5)$ \\
\hline $2(6-10 \%)$ & $13(16.8)$ & $2(8.0)$ & $15(14.7)$ \\
\hline $3(11-20 \%)$ & $11(14.3)$ & $1(4.0)$ & $12(11.8)$ \\
\hline $4(21-100 \%)$ & $5(6.5)$ & $0(0.0)$ & $5(4.9)$ \\
\hline Ki-67 & \multicolumn{3}{l|}{} \\
\hline $0(0 \%)$ & $19(24.6)$ & $19(76.0)$ & $38(37.4)$ \\
\hline $1(1-5 \%)$ & $41(53.3)$ & $5(20.0)$ & $46(45.1)$ \\
\hline $2(6 \mathrm{v} 10 \%)$ & $12(15.6)$ & $0(0.0)$ & $12(11.6)$ \\
\hline $3(11-20 \%)$ & $3(3.9)$ & $1(4.0)$ & $4(3.9)$ \\
\hline $4(21-100 \%)$ & $2(2.6)$ & $0(0.0)$ & $2(2.0)$ \\
\hline
\end{tabular}

IHC - immunohistochemical, IHC scores were estimated as described in Material and methods

distribution of the studied markers in both studied groups was summarized in Table 1 . We noted a significantly higher PR expression in GD as compared to ND $(1.23 \pm 1.31$ vs. $0.56 \pm 0.82 ; \mathrm{p}=0.0276$; Figure $2 \mathrm{~A})$. Thyrocytes affected in the GD exerted also higher proliferation rate in comparison to $\mathrm{NG}$ as measured by the expression of the Ki-67 antigen $(1.07 \pm 0.89$ vs. 0.32 $\pm 0.69 ; \mathrm{p}<0.0001 ;$ Figure 2B). No differences were noted between the two groups regarding the ER expression (GD: $1.00 \pm 1.19$ vs. ND: $0.92 \pm 0.81 ; p=0.7349$ ). 

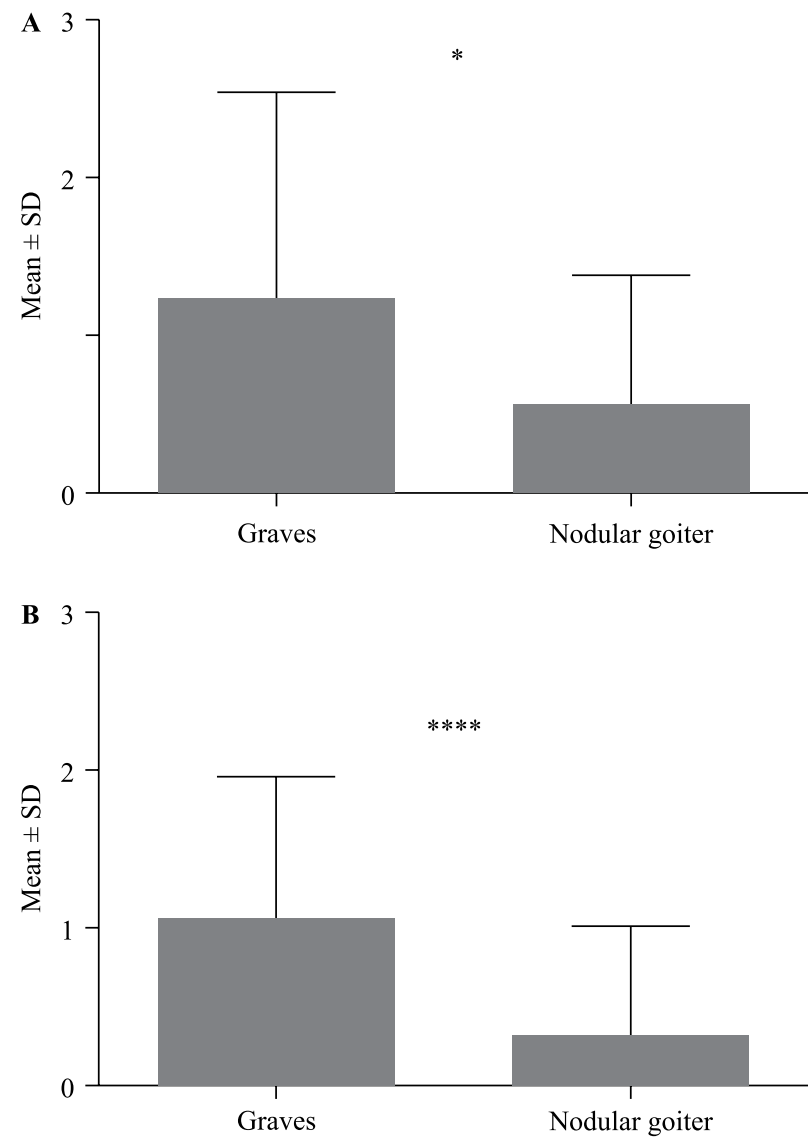

Figure 2. Differences in the expression of PR (A) and Ki-67 antigen (B) between the Graves' disease and nodular goiter patients. ${ }^{*} \mathrm{p}<0.05 ; * * * \mathrm{p}<0.0001$ (Mann-Whitney test)

No significant correlations were noted between the studied markers and patients age in the particular study groups and when both groups were analyzed together (Spearman correlation test). Expression intensities of the studied markers did not differ regarding patients age and sex in GD and NG lesions. Moreover, no differences in patients age and sex were observed between the two analyzed lesions.

\section{Discussion}

In this study we have investigated the association between expression of ER and PR in patients with GD to determine whether these factors may have influence on the proliferation of thyrocytes and overall higher incidence of GD in women. We found higher level of PR expression in thyrocytes of GD patients in comparison to NG patients. Moreover, we noted a significantly higher expression of the Ki-67 antigen in GD lesions as compared to the NG.

Cellular responses to sex hormones are dependent on the expression of hormone receptors that include two types of ER, PR and androgen receptors. $\mathrm{Cu}-$ rrently, the data concerning the expression of these receptors in thyrocytes of GD remain obscure. In women progesterone plays an important role during pregnancy. Its elevated levels keep the uterus in complete quiescence, acting through its nuclear PR [14]. Progesterone is also known for its anti-inflammatory role in human myometrial cells, as it acts antagonistically to NF- $\kappa$ B activation and induces cyclooxygenase 2 expression, the crucial enzyme in prostaglandin production and inflammation [14]. Therefore, progesterone besides its endocrine effects in pregnancy, acts as an 'immunosteroid' with local immunosuppressant functions [15]. Progesterone acts through PR via the induction of Progesterone Induced Blocking Factor (PIBF), which in turn may affect B lymphocytes. In result, the interleukin expression profile of these cells switches to a more noncytotoxic, therefore supporting the immunosuppressive role of progesterone and the PR [16].

Interestingly, in our study we observed the increased expression of PR receptors in GD patients as compared to NG patients. PR expression was also studied in rheumatoid arthritis (RA), a disease with higher prevalence in women [17]. Ishizuka et al. [17] found a significant difference in the relative expression levels of progesterone receptor type B (PR-B) in synovial tissue. In light of those results, it seems that elevated PR in thyrocytes of GD patients may represent a protective, immunosuppressive mechanism in this disease, but further research should be undertaken with inclusion of patients detailed clinical data to clarify such a hypothesis.

Two types ER are recognized, the estrogen receptor $\alpha(\mathrm{ER} \alpha)$ encoded by ESR1 gene (locus 6q25.1) and estrogen receptor $\beta(\mathrm{ER} \beta)$, which is encoded by the ESR2 gene (locus 14q23), which mediate actions of estrogen in multiple tissues [1]. The ESR2 gene is situated close to the GD locus at $14 q 31[3,18]$. In our study we estimated the expression of $\operatorname{ER} \alpha$, however, we failed to note any significant differences in its expression in GD and NG. The ER $\alpha$ was shown to promote proliferation in the tissue of the uterus, mammary gland and cells of the immune system [4, 19], therefore we analyzed its expression in GD thyroids. Until now, only one study analyzed the relationship between the ER in thyroid cells of 40 GD patients and compared to its expression noted in 15 control cases encompassing healthy thyroid tissues [20]. In contrast to the results obtained by us on a larger patients cohort, Wang et al. found a significant correlation between ER $\alpha$ expression in GD thyrocytes as compared to normal thyroid [20]. Although in our study we did not noted any significant differences in ER expression between GD and NG, we may not directly compare our results with the results obtained 
by Wang et al. as the ER expression of ER was not studied in NG lesions [20]. The results of studies analyzing ER expression in NG were inconsistent, as some studies reported even higher ER expression as this noted in our studies, whereas some did not report ER $\alpha$ expression in thyrocytes [21-23]. Studies aimed at examining the associations between ER and GD found, that the ESR2 gene was not associated with GD incidence in the Japanese population [24], whereas in the Polish study of Kisiel et al., the authors found a significant association between this gene and GD susceptibility [1]. To note, these studies were performed on isolated blood cells [1,24], whereas in our study performed on thyroid sections of GD and NG patients the $\mathrm{ER} \alpha$ expression was associated neither with patient sex nor age.

Lines of evidence suggest that pathogenesis of autoimmune diseases may involve a specific effect of sex steroid hormones on the immune system [5]. The effects of steroid hormones on immune system are heterogeneous. They may act as immunosuppressors as well as immunostimulators, and the balance between these two processes seems to be dependent upon steroid hormones levels. The higher incidence of AID in women seems to be linked to influence of sex steroid hormones on autoimmunity, as ER and PR were reported to be expressed also on some types of immune cells $[5,25,26]$. This may partly explain the higher incidence of AID noted in woman. Moreover, women have more vigorous cellular and humoral immune reactions than men [28]. Receptor-mediated effects of steroids on immune system are not well elucidated. Generally, estrogens are regarded as enhancers of immune response whereas androgens and progestins have an immunosuppressive effect [5]. Sex hormones were shown to play a role in autoimmune diseases by the modulation of cell proliferation and cytokine production of various cell types, including the cells of the immune systems [4, 19,27]. Our study suggests that the expression of ER and PR in thyrocytes of GD patients has no impact on the proliferation of thyrocytes as measured by the expression of the Ki-67 antigen, despite significantly higher expression of this proliferation marker in GD patients as compared to NG patients. However, the role of ER and PR expression in immune cells of GD patients remains to be elucidated, as this study addressed only the issue of their expression in thyrocytes, but not the surrounding immune cells.

In summary, we showed that ER and PR expression in thyrocytes of GD patients plays a limited role in the pathogenesis of this disease. Moreover, the expression of ER and PR in GD and NG patients had no impact on the proliferation of thyrocytes.

\section{Acknowledgement}

This study was supported by the grant of Wroclaw Medical University, No. ST-654.

The authors thank Mrs. Aleksandra Piotrowska, Mrs. Bozena Przygodzka, Mrs. Agnieszka Baranska and Mrs. Elzbieta Polejko from the Department of Histology and Embryology, Medical University of Wroclaw for their technical support.

\section{References}

1. Kisiel B, Bednarczuk T, Kostrzewa G et al. Polymorphism of the oestrogen receptor beta gene (ESR2) is associated with susceptibility to Graves' disease. Clin Endocrinol (Oxf). 2008;68:429-434.

2. Estienne V, Duthoit Ch, Reichert $\mathrm{M}$ et al. Androgen-dependent expression of Fc RIIB2 by thyrocytes from patients with autoimmune Graves' disease: a possible molecular clue for sex dependence of autoimmune disease. FASEB J. 2002;16:1087-1092.

3. Tomer Y, Ban Y, Concepcion E et al. Common and unique susceptibility loci in Graves' and Hashimoto diseases: results of whole-genome screening in a data set of 102 multiplex families. Am J Hum Gen. 2003;73:736-747.

4. Cutolo M, Sulli A, Seriolo B et al. Estrogens, the immune response and autoimmunity. Clin Exp Rheuma. 1995;13:217226.

5. Darnall BD, Suarez EC. Sex and gender in psychoneuroimmunology research: Past, present and future Brain Behav Immun. 2009;23:595-604.

6. Brown DC, Gatter KC. Ki67 protein: the immaculate deception? Histopathology. 2002;40:2-11.

7. Honegger J, Prettin C, Feuerhake F, Petrick M, Shulute-Monting $\mathrm{J}$ and Reincke $\mathrm{M}$ : Expression of ki-67 antigen in nonfunction- ing pituitary adenomas: Correlation with growth velocity and invasiveness. J Neurosurg. 2003;99:674-679.

8. Losa M, Barzaghi RL, Mortini P et al. Determination of the proliferation and apoptotic index in adrenocorticotropin-secret- ing pituitary tumors: comparison between micro- and macroad- enomas. Am J Pathol. 2000;156:245-251.

9. Mastronardi AL, Guiducci A, Puzzilli F. Lack of correlation between ki-67 labeling index and tumor size of anterior pituitary adenomas. BMC Cancer. 2001;1:12.

10. Salehi F, Agur A, Scheithauer BW et al. Ki-67 in pituitary neoplasms: a review-part I. Neurosurgery 2009;65:429-437.

11. Wojnar A, Kobierzycki C, Krolicka A et al. Correlation of $\mathrm{Ki}-67$ and MCM-2 proliferative marker expression with grade of histological malignancy $(\mathrm{G})$ in ductal breast cancers. Folia Histochem Cytobiol. 2010;48:442-446.

12. Wojnar A, Pula B, Piotrowska A et al. Correlation of intensity of MT-I/II expression with $\mathrm{Ki}-67$ and MCM-2 proteins in invasive ductal breast carcinoma. Anticancer Res. 2011;31: 3027-3033.

13. Luporsi E, André F, Spyratos F et al. Ki-67: level of evidence and methodological considerations for its role in the clinical management of breast cancer: analytical and critical review. Breast Cancer Res Treat. 2012;132:895-915.

14. Hardy DB, Janowski BA, Corey DR et al. Progesterone receptor plays a major antiinflammatory role in human myometrial cells by antagonism of nuclear factor-kappaB activation of cyclooxygenase 2 expression. Mol Endocrinol. 2006;20:2724-2733. 
15. Arck P, Hansen PJ, Jericevic. BM et al. Progesterone during pregnancy: Endocrine- immune cross talk in mammalian species and role of stress. Am J Reproduct Immunol. 2007;58:268-279.

16. Druckmann R, Druckman MA. Progesterone and the immunology of pregnancy. J Steroid Biochem Mol Biol. 2005;97: 389-396.

17. Ishizuka M, Hatori $M$, Suzuki $T$ et al. Sex steroid receptors in rheumatoid arthritis. Clin Sci (Lond). 2004;106:293-300.

18. Tomer Y, Barbesino G, Keddache $M$ et al. Mapping of a major susceptibility locus for Graves' disease (GD-1) to chromosome 14q31.J Clin Endocrinol Metab. 1997;82:1645-1648.

19. Morani A, Warner M, Gustafsson JA. Biological functions and clinical implications of oestrogen receptors alfa and beta in epithelial tissues. J Intern Med. 2008;264:128-142.

20. Wang S, Mao S, Zhao G et al. Relationship between estrogen receptor and Graves' disease. Zhonghua Wai Ke Za Zhi. 2000;38:619-621.

21. Ceresini G, Morganti S, Graiani V et al. Estrogen receptor (ER)-beta, but not ER-alpha, is present in thyroid vessels: immunohistochemical evaluations in multinodular goiter and papillary thyroid carcinoma. Thyroid. 2006;16:1215-1220.
22. Arain SA, Shah MH, Meo SA, Jamal Q. Estrogen receptors in human thyroid gland. An immunohistochemical study. Saudi Med J. 2003;24:174-178.

23. Hiasa $Y$, Nishioka $H$, Kitahori $Y$ et al. Immunohistochemical analysis of estrogen receptors in 313 paraffin section cases of human thyroid tissue. Oncology. 1993;50:132-136.

24. Ban Y, Tozaki T, Taniyama M, et al. Lack of association between estrogen receptor beta dinucleotide repeat polymorphism and autoimmune thyroid diseases in Japanese patients. BMC Med Genet. 2001;2:1.

25. Nancy P, Berrih-Aknin S. Differential estrogen receptor expression in autoimmune myasthenia gravis. Endocrinology. 2005;146, 2345-2353.

26. Dziedziejko V, Kurzawski M, Safranow K et al. Oestrogen receptor polymorphisms in female patients with rheumatoid arthritis. Scand J Rheumatol. 2011;40:329-333.

27. Doukas C, Saltiki K, Mantzou A et al. Hormonal parameters and sex hormone receptor gene polymorphisms in men with autoimmune diseases. Rheumatol Int. 2013;33:575-582.

28. Bouman A, Heineman MJ, Faas MM. Sex hormones and the immune response in humans. Hum Reprod Update. 2005;11:411-423. 